\title{
Gamma-rays and neutrinos from accreting neutron stars
}

\section{Włodek Bednarek*}

Department of Astrophysics, University of Łódź, ul. Pomorsla 149/153, 90-236 Łódź, Poland E-mail: bednareastro.phys.uni.lodz.pl

\begin{abstract}
The matter from a star can be partially captured by a companion neutron star (NS) creating a very turbulent and magnetized transition region at some distance from the NS surface. We consider the consequences of particle acceleration at such transition region. Accelerated electrons lose energy on the synchrotron process and the inverse Compton (IC) scattering of thermal radiation from the NS surface and/or the companion star. We calculate the synchrotron spectra (from X-rays to soft $\gamma$-rays) and $\gamma$-ray IC spectra in the case of sources accreting the matter under the accretor and propeller scenarios. Depending on the accretion stage, the $\mathrm{GeV}$ and/or the $\mathrm{TeV} \gamma$-ray emission can be produced. The model can explain the $\mathrm{TeV} \gamma$-ray emission from recently discovered massive binary system (LSI $303+61$ ). Moreover, it predicts $\mathrm{GeV} \gamma$-ray fluxes from some compact X-ray binary systems detectable by the Fermi-LAT telescope. Hadrons accelerated in such scenario can interact with the radiation from the NS surface producing $\gamma$-rays and neutrinos. We estimate the expected neutrino fluxes from some example binary systems in terms of this model which might be observable by the IceCube neutrino telescope.
\end{abstract}

The Extreme sky: Sampling the Universe above $10 \mathrm{keV}$ - extremesky2009,

October 13-17, 2009

Otranto (Lecce) Italy

${ }^{*}$ Speaker. 


\section{Introduction}

Compact binary systems containing neutron stars are strong sources of X-ray emission which is produced as a result of accretion of matter from a classical star onto the NS. The accretion process can occur in different stages depending on the parameters of the NS (surface magnetic field, rotational period) and the accretion rate. Such sources have been expected to accelerate particles to relativistic energies which interacting with the matter and radiation should produce high energy $\gamma$-rays and neutrinos. In fact, the matter accreting onto the NS interacts with its rotating magnetosphere creating good conditions for acceleration of particles. The aim of this paper is to investigate possible high energy radiation produced by these energetic particles interacting with the radiation from the NS surface and the companion star.

\section{The Model}

We consider a compact binary system containing rotating neutron star (NS) and a classical star. It is assumed that the mass from the companion star is effectively captured by a strong gravitational potential of the NS. Depending on the rotational period and surface magnetic field of NS, the accretion process onto NS can occur in the phase of accretor, propeller, or ejector. A very turbulent and magnetized transition region is formed when the magnetic pressure balances the pressure of infalling matter. In the case of accretor, most of the matter falls onto the NS surface creating a small hot region on the NS surface (for details of this scenario see [1, 2, 3).

The distance of the transition region from the surface of the neutron star can be estimated by comparing the energy density of the magnetic field with the energy density of accreting matter. It is

$$
R_{\mathrm{A}}=4 \times 10^{8} B_{12}^{4 / 7} M_{16}^{-2 / 7} \mathrm{~cm},
$$

where the magnetic field at the neutron star surface is $B_{\mathrm{NS}}=10^{12} B_{12} \mathrm{G}$ and the accretion rate is $\dot{M}_{\mathrm{acc}} \approx 10^{16} M_{16} \mathrm{~g} \mathrm{~s}^{-1}$.

The magnetic field at $R_{\mathrm{A}}$ is estimated assuming its dipole structure in the inner magnetosphere

$$
B_{\mathrm{A}}=1.6 \times 10^{4} M_{16}^{6 / 7} B_{12}^{-5 / 7} \quad \mathrm{G} .
$$

The matter penetrate below the light cylinder radius for the rotational periods of NS (from comparison of the light cylinder radius, $R_{\mathrm{L}}=c P / 2 \pi$, with $R_{\mathrm{A}}$ ),

$$
P_{\mathrm{I}}>0.084 B_{12}^{4 / 7} M_{16}^{-2 / 7} \quad \mathrm{~s} .
$$

The matter can reach the NS surface (accretor phase) when the rotational velocity of the NS magnetosphere is lower than the keplerian velocity of the matter at $R_{\mathrm{A}}$. This happens for the periods,

$$
P_{\mathrm{II}}>3.7 B_{12}^{6 / 7} M_{16}^{-3 / 7}
$$

The accretion occurs in the propeller phase (matter stopped at some distance from NS surface) for $P_{\mathrm{I}}<P<P_{\mathrm{II}}$. 
Particles (electrons and hadrons) can gain energy from the acceleration mechanism at a rate which can be parametrised by,

$$
\dot{P}_{\mathrm{acc}}=\xi c E_{p} / r_{\mathrm{L}} \approx 2.6 \times 10^{4} \xi_{-1} M_{16}^{6 / 7} B_{12}^{-5 / 7} \mathrm{erg} \mathrm{s}^{-1},
$$

where $\xi=10^{-1} \xi_{-1}$ is the acceleration parameter, $R_{\mathrm{L}}=E_{p} / e B_{\mathrm{A}}$ is the Larmor radius, and $E_{p}$ is the energy of particle.

Electrons lose energy on the IC process in the Thomson (T) and the Klein-Nishina (KN) regimes. In the accretor stage, when the matter reachs the NS surface and emits thermal soft radiation from a polar cap region, the energy density of these thermal photons at the acceleration region above the NS surface is,

$$
\rho_{\text {cap }} \approx 1.4 \times 10^{8} M_{16}^{11 / 7} B_{12}^{-8 / 7} \mathrm{erg} \mathrm{cm}^{-3} .
$$

The radius of the polar cap region on the NS surface can be estimated on, $R_{\text {cap }}=\left(R_{\mathrm{NS}}^{3} / R_{\mathrm{A}}\right)^{1 / 2} \approx 5 \times$ $10^{4} B_{12}^{-2 / 7} M_{16}^{1 / 7} \mathrm{~cm}$, and its surface temperature is, $T_{\text {cap }}=\left(L_{\mathrm{X}} / \pi R_{\text {cap }}^{2} \sigma\right)^{1 / 4} \approx 4.7 \times 10^{7} B_{12}^{1 / 7} M_{16}^{5 / 28} \mathrm{~K}$. The energy density of the magnetic field at $R_{\mathrm{A}}$ is, $\rho_{\mathrm{B}}=B_{\mathrm{A}}^{2} / 8 \pi \approx 10^{7} M_{16}^{12 / 7} B_{12}^{-10 / 7} \mathrm{erg} \mathrm{cm}^{-3}$. The energy losses of electrons for the synchrotron and the IC in the T regime can be calculated from,

$$
\dot{P}_{\text {loss }}=(4 / 3) c \sigma_{\mathrm{T}} \rho \gamma^{2} \approx 2.7 \times 10^{-14} \rho_{(\mathrm{cap}, \mathrm{B}, \star)} \gamma^{2} \mathrm{erg} \mathrm{s}^{-1} .
$$

By balancing energy gains and synchrotron losses we get the maximum Lorentz factors for electrons,

$$
\gamma_{\max } \approx 3 \times 10^{5} \xi_{-1}^{1 / 2} B_{12}^{5 / 14} M_{16}^{-3 / 7} .
$$

We conclude that electrons can reach energies of the order of $\sim 100 \mathrm{GeV}$ in the case of classical NS. However, the energies can become of the order of $\sim \mathrm{TeV}$ for the NS with the surface magnetic field typical for magnetars.

The maximum energies of the accelerated hadrons are estimated by the balance between the acceleration time scale and the time scale which defines the confinement of hadrons inside the turbulent region. The lower limit on this last time scale gives the escape time scale of hadrons from the acceleration site. It can be defined as, $\tau_{\mathrm{esc}}=R_{\mathrm{A}} / v_{\mathrm{f}} \approx 0.4 B_{12}^{6 / 7} M_{16}^{-3 / 7} \mathrm{~s}$, where $v_{\mathrm{f}}=$ $\left(2 G M_{\mathrm{NS}} / R_{\mathrm{A}}\right)^{-1 / 2}$ is the free fall velocity of matter at $R_{\mathrm{A}}\left(\mathrm{G}\right.$ - gravitational constant and $M_{\mathrm{NS}}$ - the mass of NS). By comparing this time scale with the acceleration time scale, $\tau_{\mathrm{acc}}=m_{\mathrm{p}} \gamma_{\mathrm{p}} / \dot{P}_{\text {acc }}$ (where $m_{\mathrm{p}}$ is the proton mass), we can estimate the Lorentz factors to which hadrons can be accelerated,

$$
\gamma_{\mathrm{p}} \approx 6 \times 10^{6} \xi_{-1} B_{12}^{1 / 7} M_{16}^{3 / 7}
$$

This estimate is of the order of the maximum possible Lorentz factor of particles, which can be accelerated within the region with characteristic diameter $R_{\mathrm{A}}$ and magnetic field $B_{\mathrm{A}}$, defined by the condition that the Larmor radius is smaller than the Alfven radius, $R_{\mathrm{L}}<R_{\mathrm{A}}, \gamma_{\mathrm{p}}^{\max } \approx$ $2 \times 10^{6} B_{12}^{-1 / 7} M_{16}^{4 / 7}$.

We can also estimate the energy losses of hadrons on collisions with thermal photons from the NS surface inside the transition region,

$$
\dot{P}_{\mathrm{p} \gamma \rightarrow \pi}=\sigma_{\mathrm{p} \gamma} c n K E_{p}
$$


where $n=n_{\mathrm{bb}}\left(R_{\text {cap }} /\left(\left(R_{\mathrm{A}}-R_{\mathrm{NS}}\right)+R_{\mathrm{cap}}\right)\right)^{2}\left(n \approx n_{\mathrm{bb}} R_{\text {cap }}^{2} / R_{\mathrm{A}}^{2}\right.$ for $\left.R_{\mathrm{A}} \gg R_{\text {cap }}\right)$ is the density of the black body photons coming from the polar cap at the distance of the transition region $R_{\mathrm{A}}$, and $\sigma_{p \gamma} \approx 3 \times 10^{-28} \mathrm{~cm}^{-2}$ and $K \approx 0.15-0.35$ are the cross section and the in-elasticity for pion production due to collision of relativistic protons with thermal photons. In our estimations we apply the average value for $K=0.25$. As above, we can estimate the maximum possible energies of accelerated hadrons due to their energy losses on pion production in collisions with thermal photons by comparing Eqs. (2.5) and (2.10). We obtain the limit on the Lorentz factor of hadrons,

$$
\gamma_{\mathrm{p} \gamma \rightarrow \pi} \approx 8 \times 10^{7} \xi_{-1} B_{9}^{4 / 7} M_{16}^{33 / 28} .
$$

These maximum Lorentz factors of hadrons are typically above the maximum Lorentz factors of hadrons estimated above based on their escape from the acceleration region especially in the case of large accretion rates. Therefore, we conclude that hadrons are at first accelerated to maximum energies allowed by the escape mechanism and after that they interact with thermal photons during their fall onto the NS surface.

It is assumed that particles are accelerated with the power law spectrum up to the maximum energies estimated above.

The maximum power transfered from the rotating NS to the accreting matter can be,

$$
L_{\mathrm{acc}}=\dot{M}_{\mathrm{acc}} v_{\mathrm{rot}}^{2} / 2 \approx 3 \times 10^{34} B_{12}^{8 / 7} M_{16}^{3 / 7} P^{-2} \mathrm{erg} \mathrm{s}^{-1} .
$$

where $v_{\text {rot }}=2 \pi R_{A} / P$ is the rotational velocity of the magnetic field of NS at $R_{\mathrm{A}}$, and $P$ is in seconds. A part, $\eta$, of this power can be used for the acceleration of particles.

\section{High energy radiation from accreting NS}

Accelerated particles interact mainly with the thermal radiation field from the polar cap on the NS surface and from the surface of the massive star. Below we calculate the high energy radiation produced by electrons and hadrons in the accretor stage and produced by electrons in the propeller stage.

\subsection{Gamma-rays}

High energy $\gamma$-rays are produced in the inverse Compton scattering process of thermal photons from the polar cap and radiation of the massive companion star. They can be also absorbed in this same radiation field. We calculate the optical depths for $\gamma$-rays assuming that they are produced by electrons in the transition region at the distance $R_{\mathrm{A}}$ from the stellar surface and interact with the thermal radiation from NS (see Fig. $3 \mathrm{in}[\mathrm{[}]$ ). The optical depths are clearly above unity for large accretion rates onto the classical NS (surface magnetic field of the order of $10^{12} \mathrm{G}$ ) and the milisecond NS (surface magnetic field of the order of $10^{9} \mathrm{G}$ ). For low accretion rates $\left(<10^{16} \mathrm{~g}\right.$ $\mathrm{s}^{-1}$ ), the optical depths for $\gamma$-ray photons are below unity for energies above $\sim 10 \mathrm{GeV}$. In this case $\mathrm{GeV}-\mathrm{TeV} \gamma$-rays can escape from the radiation field of the polar cap without significant absorption. However, they can be additionally absorbed if the radiation of the companion star is strong enough. We consider $\gamma$-ray production by electrons in the accretor scenario (for classical pulsar) and the propeller scenario (for the strongly magnetized NS, so called magnetar). 


\subsubsection{Accretor scenario for classical pulsar}

Due to the large optical depths, $\gamma$-rays are produced in the IC $e^{ \pm}$pair cascade initiated by relativistic electrons in the radiation of the polar cap (for details of the the cascade scenario see e.g. [1]. 凹). In order to follow the process of $\gamma$-ray production, we developed the numerical code which simulate the cooling process of electrons taking into account not only the $\gamma$-ray production in the IC process but also the synchrotron energy losses of primary electrons and secondary cascade $e^{ \pm}$pairs. Two models for injection of relativistic electrons are considered: (1) injection with the power law spectrum up to the maximum Lorentz factor $\gamma_{\max }$, and (2) monoenergetic with the Lorentz factors $\gamma_{\max }$. We investigate two general scenarios describing the cases of pure accretor phase and the intermediate accretor-propeller phase. The example spectra are shown for different values of $\dot{M}, \xi$ and $B$ [四]. They are above the sensitivity of the Fermi-LAT telescope. Therefore, we predict that the Fermi-LAT telescope have a chance to detect $\sim 0.1-1 \mathrm{GeV} \gamma$-ray emission from some compact binary systems. In more detail we discuss the case of the hard X-ray massive binary system IGR J19140+0951 recently discovered by INTEGRAL, predicting predicting its detection for specific parameters of the accretion scenario and acceleration model of particles [1].

\subsubsection{Propeller scenario for magnetar}

In this scenario, a neutron star with the superstrong magnetic field accretes the matter in the propeller scenario, i.e. the matter is stopped at some distance from the NS and accelerated electrons interact with the magnetic field at the transition region and radiation from the massive companion star. Most of the matter is rather expelled from the vicinity of NS in this case starting perpendicular outflows similar to the jet features observed in some type of binary systems called microquasars. The $\gamma$-rays are produced by electrons in the anisotropic IC $e^{ \pm}$pair cascade process occurring in the massive star radiation. As an example, we consider the TeV $\gamma$-ray binary system, LSI +61303 , which was suggested to contain a magnetar. The calculations are compared with the X-ray to $\mathrm{TeV}$ $\gamma$-ray spectrum from this source [2]. It is concluded that such accreting magnetar scenario can be responsible for $\mathrm{TeV} \gamma$-ray emission from LSI +61303 at the apastron passage.

\subsection{Neutrinos}

We calculate the spectra of muon neutrinos coming from the decay of pions produced in collisions of hadrons with the thermal radiation from the polar cap on the NS surface. In this case, two types of hadron injection spectra are considered: (a) a power law differential spectrum with the spectral index 2.1 which cuts at energies estimated by above, and (b) a mono-energetic spectrum with these maximum energies. For the example calculations, we fix the following parameters describing acceleration of hadrons: $\xi_{-1}=0.1$, and $\eta=0.1$. We calculate the muon neutrino event rates that are expected in a $\mathrm{km}^{2}$ neutrino detector from millisecond pulsars (MSPs) at the distance of $5 \mathrm{kpc}$ which accrete matter from the stellar wind [3]. The typical parameters for the MSPs and the accretion rates are considered. The number of neutrino events is estimated by integrating the muon neutrino spectra over the probability of their detection,

$$
N_{\mu}=\frac{S}{4 \pi D^{2}} \int_{E_{v}^{\min }}^{E_{v}^{\max }} P_{v \rightarrow \mu}\left(E_{v}\right) \frac{d N_{v}}{d E_{v} d t} d E_{v}
$$


where $S=1 \mathrm{~km}^{2}$ is the surface of the detector, $D$ is the distance to the source, $P_{v \rightarrow \mu}\left(E_{v}\right)$ is the energy dependent detection probability of a muon neutrino [5], $d N_{v} / d E_{v} d t$ is the differential neutrino spectrum produced at the source, $E_{v}^{\min }=10^{2} \mathrm{GeV}$ is the minimum energy of produced neutrinos over which we integrate the event rate in the $1 \mathrm{~km}^{2}$ neutrino telescope and the maximum energy of neutrinos produced in this model is typically in the range $E_{v}^{\max }=10^{4}-10^{5} \mathrm{GeV}$. This maximum energy of neutrinos can not be simply expressed since it depends on the parameters of the model (see above). Therefore, it is obtained numerically in our calculations. The expected neutrino event rates from millisecond pulsars range from a few up to several per $\mathrm{km}^{2}$ per $\mathrm{yr}$ (depending on the model). These event rates should be detected by the IceCube neutrino detector. Note that several up to a hundred of milisecond pulsars are expected inside typical globular cluster. Therefore, we also suggest that globular clusters can produce detectable sources of neutrinos for the large scale neutrino telescopes (IceCube, KM3NET).

We also calculate the neutrino event rates expected from two nearby X-ray pulsars inside the binary systems GROJ $1744-28$ and A $0535+262$. They are characterized by long rotational periods, strong surface magnetic fields, and high accretion rates. Also from these sources a few neutrino events per year are expected in an IceCube size neutrino detector.

\section{Conclusion}

We calculated the expected X-ray to $\gamma$-ray and neutrino emission from the rotating neutron stars which accrete the matter in the terms of the accretor and propeller scenarios. The $\gamma$-ray emission, expected in such a model, has been calculated the high mass X-ray binary systems: IGR J19140+0951, discovered by the INTEGRAL and the TeV $\gamma$-ray binary LSI +61 303. We conclude that compact massive binaries recently discovered by the INTEGRAL are likely to be detected in the $\gamma$-rays by the Fermi-LAT telescope. The accreting magnetar scenario can be also responsible for the TeV $\gamma$-ray emission from LSI +61 303.

We also estimated the neutrino event rates in the $\mathrm{km}^{2}$ detector for two relatively nearby binary systems which accrete at large rate, GRO 1744-2 and A 0535+262. Other considered sources are expected to emit lower neutrino fluxes due to the larger distances or lower accretion rates. We also estimate the neutrino event rates expected from the millisecond pulsars at the typical distance of globular clusters. We conclude that some accreting millisecond pulsars could become detectable neutrino sources. The neutrino event rates expected from the whole population of MSPs in a specific globular cluster can be enhanced due to the cumulative contribution from many milisecond pulsars.

\section{References}

[1] Bednarek, W. 2009 A\&A 495, 919

[2] Bednarek, W. 2009 MNRAS 397, 1420

[3] Bednarek, W. 2009 PRD 79, 3010

[4] Bednarek, W. 2000 A\&A 363, 646

[5] Gaisser, T.K., Grillo, A.F. 1987 Phys. Rev. D 39, 1481 